\title{
Shift Invariant Degradation Analysis and Restoration Method for Photograph Degraded by Rectilinear Smear
}

\author{
W. Fawwaz Al Maki, T. Kitagawa, T. Hori, and S. Sugimoto \\ Dept. of Electrical and Electronic Engineering, Ritsumeikan University \\ 1-1-1 Noji-Higashi, Kusatsu City, Shiga 525-8577, Japan \\ E-mail: sugimoto@se.ritsumei.ac.jp
}

\begin{abstract}
The photographs acquired by an optical system are sometimes degraded by relative motion between an imaging system and the original scene during image formation. Traditional methods assume that the relative motion that causes rectilinear smear on the photographs happens at a constant velocity. However, in practical situation, accelerated motion can cause rectilinear smear on the photograph and is the general form of the linear motion. In this paper, we present an analysis of rectilinear smear due to the accelerated motion and the restoration of photographs degraded by the rectilinear smear. The blurred image is restored by using an inverse filter. Experimental results show the feasibility and reliability of the method.
\end{abstract}

\section{Introduction}

Many computer vision algorithms assume that there are no relative motion between an imaging equipment and an object during the process of capturing the object. This assumption is not true since the relative motion often exists in the imaging process. Relative motion between the camera and the captured object during exposure time can cause the resulted photograph to be degraded by rectilinear smear. Rectilinear smear is a form of bandwidth reduction due to imperfect image formation proces. The relative motion between the original scene and an imaging equipment during the acquisition process could be the constant velocity motion or the accelerated motion.

Removing rectilinear smear from a photograph is important in the practical point of view. Therefore, many researchers have proposed various algorithms to overcome this problem $[1,2,3,4,5,6,7,8]$. However, the researchers assumed that relative motion happens at a constant velocity. In the case of constant velocity motion, the point spread function (PSF) is a window function. Estimation of PSF parameters can be per- formed by analyzing the window function in frequency domain. The blurred image can be restored by using any spatial domain-based or frequency domain-based image restoration techniques. The frequency domain based-image restoration method was used in [7]. In [9], a spatial domain restoration method that removed motion blur along the motion blur direction gave satisfying results.

However, in the real application, the captured image is often blurred by the accelerated motion. The accelerated motion case is an interesting problem seen from the practical point of view and is more realistic than that of the constant velocity motion case. Also, the accelerated linear motion blur is the general form of the linear motion blur.

In many applications, it is often required to remove the motion blur from an image by using available image restoration techniques. Image restoration is one of the fundamental problems in image processing $[10,11,12]$. The purpose of image restoration is to obtain a sharp image from a given blurred image. Restoration of an image degraded by the linear motion is challenging since it has wide applications $[6,13]$.

In this paper, the linear motion blurred image that we consider is that occurs if a camera held by an accelerated vehicle captures a scene. Therefore, the recorded image is uniformly blurred by the relative motion between the camera and the object. Such blurred image can also occur if handshake happens when a camera captures a scene or an object.

Restoration of the linear motion blurred images depends on the degradation process model and the point spread function model. In this paper, degradation process is modeled as a linear shift invariant system. Therefore, an accelerated linear motion blurred image is expressed as a convolution product between the true image and the PSF. The accelerated linear motion blurred image restoration is then an inverse problem. Here, the sharp image can be obtained by applying an inverse filter to the blurred image. 
To obtain the sharp image from the blurred image, the PSF of the accelerated linear motion is important to model. The PSF can be modeled based on its physical phenomena. The parameters of the PSF are motion length, motion direction, and acceleration factor. In this paper, the PSF model is given for horizontal motion case. However, the PSF model can easily generalized to the case where the motion direction can be any angle.

In this paper, the image restoration technique is presented for known PSF parameters. The idea presented in [9] can be adopted to remove the accelerated motion blur. In the blind image restoration problem, the PSF parameters must be estimated from the blurred image. Then, image restoration is performed after estimating the PSF parameters.

\section{Shift Invariant Degradation Analysis}

\subsection{Analysis of Accelerated Linear Motion}

Let a sensor be located at camera coordinate $(x, y)$ and an object be located at its coordinates $(u(t), w(t))$. The total exposure of an image is obtained by integrating the instantaneous exposure over the time interval during the shutter is open. Therefore, if the object translates from its position, image of the moving object recorded by the sensor during a finite exposure time $T$ is given by

$$
g(x, y)=\frac{1}{T} \int_{0}^{T} f(u(t), w(t)) \mathrm{d} t
$$

where $g(x, y)$ is the blurred image and $f(u(t), w(t))$ is the original image. Here, rectilinear smear will apper on the image due to the linear motion recorded by the camera. The aforesaid formula is similar to that given in $[14]$.

The linear motion can happen at a constant velocity or a variably velocity. In this paper, the case of the linear motion with a constant acceleration is discussed. We assume that an object undergoes a planar motion in one direction. If the object translates with an initial velocity $V_{0}$ and a constant acceleration $a$ along the $\mathrm{x}$ axis, its position in the object coordinates at time $t$ is given by

$$
\begin{aligned}
& w(t)=y, \\
& u(t)=x-s(t)=x-V_{0} t-\frac{1}{2} a t^{2} .
\end{aligned}
$$

By using (3) and the following relations:

$$
\begin{aligned}
s(t) & =V_{0} t+\frac{1}{2} a t^{2} \\
a & =\left(V_{t}-V_{0}\right) / t
\end{aligned}
$$

we can rewrite (1) as

$$
g(x, y)=\frac{1}{T} \int_{x-V_{0} T-\frac{1}{2} a T^{2}}^{x} f(u, y) \frac{1}{\sqrt{V_{0}^{2}+2 a(x-u)}} \mathrm{d} u .
$$

We assume that the degradation process due to the accelerated linear motion is shift linear invariant. Therefore, a photographic image degraded by the rectilinear smear can be expressed as a product of convolution between the original image and the PSF:

$$
\begin{aligned}
g(x, y) & =f(x, y) \circledast h(x, y) \\
& =\int_{-\infty}^{\infty} \int_{-\infty}^{\infty} f(u, w) h(x-u, y-w) \mathrm{d} u \mathrm{~d} w
\end{aligned}
$$

Since the linear motion happens in the horizontal direction, (7) is expressed as

$$
g(x, y)=g_{y}(x)=\int_{-\infty}^{\infty} f(u, w) h(x-u) \mathrm{d} u .
$$

\subsection{Point Spread Function}

In the case of uniform linear motion blurred image, PSF is a rectangular function given by

$$
h(x, y)= \begin{cases}1 / L & \text { if } 0 \leq|x| \leq L \cos \theta ; y=L \sin \theta \\ 0 & \text { otherwise }\end{cases}
$$

where $L$ is the motion length given by

$$
L=V t_{e} .
$$

In (10), $V$ is the constant velocity and $t_{e}$ is the exposure time. Eq. (10) does not hold for the accelerated linear motion case in the accelerated motion, the velocity parameter will change over time or as the acceleration factor gives influence. If $z$ is the displacement, the velocity in the acceleration motion is given by

$$
\tilde{V}(z)=\sqrt{V_{0}^{2}+2 a z} ; \quad 0<z<L
$$

The PSF of the accelerated linear motion can be obtained from analysis of the shift invariant degradation process. From (5) and (8), and by using $z=x-u$, the PSF of rectilinear smear due to the constant acceleration motion is written as

$$
h(z)= \begin{cases}\frac{1}{T \sqrt{V_{0}^{2}+2 a z}} & \text { for } 0 \leq z \leq L \\ 0 & \text { otherwise }\end{cases}
$$

Eq. (12) exhibits characteristic of the PSF of the accelerated linear motion blur. The PSF of the accelerated motion is influenced by the initial velocity $V_{0}$ and 
the constant acceleration $a$. By using (11), (12) can be rewritten as

$$
h(z)= \begin{cases}\frac{1}{T \tilde{V}(z)} & \text { for } 0 \leq z \leq L \\ 0 & \text { otherwise }\end{cases}
$$

A relation between the constant velocity and the acceleration can be written as [15]:

$$
R=\frac{V_{0}^{2}}{a} \text {. }
$$

In the aforesaid formula, $R$ is the acceleration factor. If $R$ is very large, i.e. $V_{0}^{2}>>a$, the PSF of rectilinear smear due to the constant acceleration motion will be close to that of the smear caused by the constant velocity motion. Although, the constant velocity dominates the characteristic of the PSF, the acceleration still has the influence. When $R$ is small, i.e. $V_{0}^{2}<<a$, the difference will be obvious. In this condition, the value of $R$ gives a significant influence on the rectilinear smear that appears on the degraded image. If (12) is normalized using $h(0)$ and (14), we get

$$
h(z)= \begin{cases}{[1+(2 z / R)]^{-\frac{1}{2}}} & \text { for } 0 \leq z \leq L \\ 0 & \text { otherwise }\end{cases}
$$

In $(15), h(z)$ values are between 0 and 1 . In this paper, we use the PSF model expressed in (15). In the blind image restoration problem where the PSF parameters are necessary to estimate, the use of the aforesaid PSF model will avoid us to estimate $V_{0}^{2}$ and $a$. Therefore, we can only estimate $R$ and the motion length $L$.

\section{Image Restoration}

Image restoration method presented here is based on the linear shift invariant degradation analysis described in the preceded section. As the rectilinear smear caused by the accelerated motion is linear shift invariant, degraded image in discrete form can be modeled as

$$
g(i, j)=\sum_{p=-P}^{P} \sum_{q=-Q}^{Q} h_{p, q} f(i-p, j-q) .
$$

In this paper, the blurred image is divided into some regions by considering the motion direction. Therefore, the blurred image matrix can be expressed as a series of vectors. Here, the 2-D degradation process is decomposed into 1-D degradation process. The objective of expressing 2-D degradation process into 1-D degradation process is to alleviate the motion blurred image restoration.
From (16), the 1-D degradation process is given by

$$
g_{i}(j)=\sum_{l=0}^{L-1} h_{l} f_{i}(j-l) .
$$

To express the degradation process in a matrix form, it is important to consider the boundary value problem. In this paper, we assume that the pixel values outside an image are zeroes. This assumption is common and reasonable in image restoration techniques. Moreover, we do not employ frequency domain-based image restoration techniques where assumption of zero boundary values can cause ringing artifacts in the restored image. From (17), by assuming the boundary values $f(i, j)=0$ for $i \notin I$ and $j \notin J$, we have

$$
\left[\begin{array}{c}
g(i, 1) \\
g(i, 2) \\
\vdots \\
\vdots \\
\vdots \\
g(i, J)
\end{array}\right]=\left[\begin{array}{cccccc}
h_{L-1} & & & & & \\
h_{L-2} & \ddots & & & 0 & \\
\vdots & \ddots & \ddots & & \\
h_{0} & \ddots & \ddots & \ddots & \\
& \ddots & \ddots & \ddots & \ddots & \\
0 & & h_{0} & \cdots & \cdots & h_{L-1}
\end{array}\right]\left[\begin{array}{c}
f(i, 1) \\
f(i, 2) \\
\vdots \\
\vdots \\
\vdots \\
f(i, J)
\end{array}\right]
$$

which can also be expressed in a matrix form as $G_{m}=$ $H F_{m}$. In the aforesaid formula, $J$ is the number of pixels in an image.

The image restoration problem will be an inverse problem to get $\hat{F}_{m}$. Therefore, inverse filtering for each blurred image vectors is written as

$$
\hat{F}_{m}=H^{-1} G_{m}
$$

Since the blurred image was decomposed into a series of vectors, the restored image is achieved by composing the vectors obtained from the above equation. Therefore, the restored image $\hat{F} \in \Re^{I \times J}$ is expressed as

$$
\hat{F}=\left[\begin{array}{c}
\hat{F}_{1}^{\mathrm{T}} \\
\hat{F}_{2}^{\mathrm{T}} \\
\vdots \\
\vdots \\
\hat{F}_{M}^{\mathrm{T}}
\end{array}\right]
$$

\section{Experimental Results and Discussion}

We implemented the PSF model to simulate the accelerated linear motion blurred image. Then, the blurred image was restored by using the method described in the preceding section. The image used in the experiments was a grayscale image. Fig. 1 shows the original image. The image was degraded by accelerated linear 
motion with the given $R$ and the motion length. Fig. 2 shows the blured image for motion length 30 pixels and $R=441$. Here, we simulated the blurred image for $V_{0}=42 \mathrm{~ms}^{-1}$ and $a=4 \mathrm{~ms}^{-2}$.

For the accelerated linear motion blurred image in the horizontal direction, the blurred image could be restored by using the inverse filter. Fig. 3 shows the restored image. The image restoration results showed noticeable improvements in the image quality. The method presented here shows its feasibility to remove the accelerated linear motion from an image.

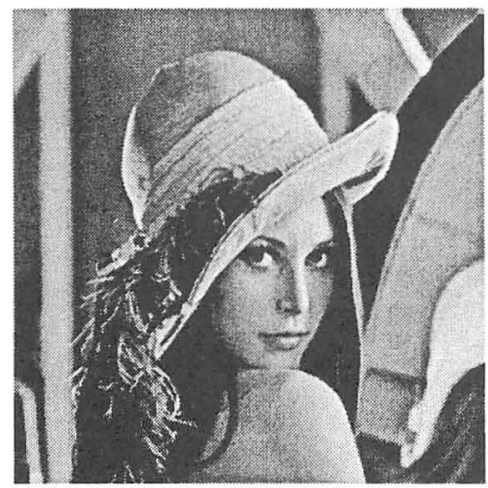

Fig. 1: Original Image

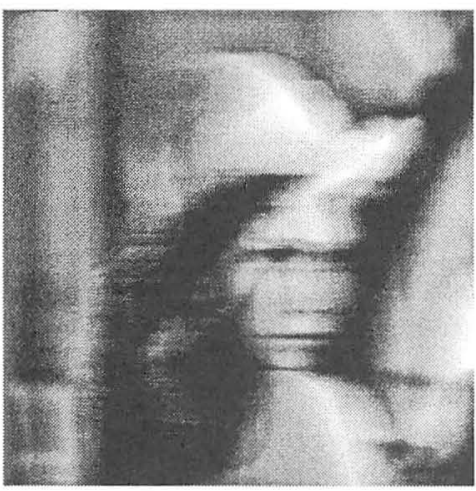

Fig. 2: Blurred Image

\section{Conclusions}

Analysis of rectilinear smear caused by the accelerated linear motion has been discussed in this paper. We consider that the degraded image is uniformly blurred. The blur happens at all regions of the image with the same direction. Therefore, mathematical model for the PSF of motion blur and the degraded image were derived by assuming that the degradation process is shift invariant.

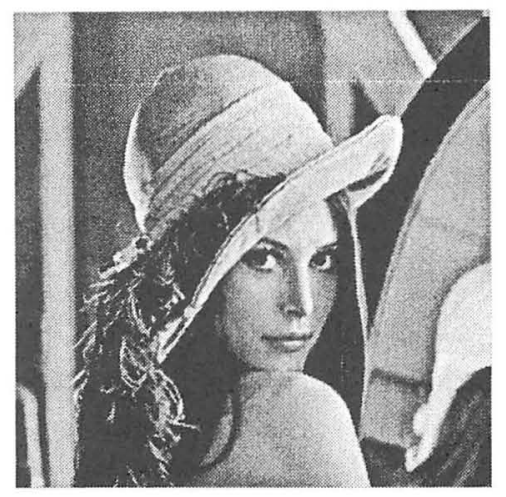

Fig. 3: Restored Image

Our restoration approach gives satisfactory results to remove the accelerated linear motion blur. The spatial domain-based image restoration technique can avoid ringing artifacts on the restored image that often happens in the frequency domain-based image restoration method. In future works, we will extend the problem to the blind image restoration problem in which the PSF parameters estimation is challenging and important. Once the PSF parameters are obtained, the blurred image can be restored by using the method discussed in this paper.

\section{References}

[1] D. Slepian: Restoration of Photographs Blurred by Image Motion, Bell Syst. Tech. J., vol. 46, pp. 2353-2363, 1967.

[2] M. M. Sondhi: Image Restoration: The Removal of Spatially Invariant Degradations, Proc. of the IEEE, vol. 60, pp. 842-853, 1972.

[3] Katayama: Restoration of Images Degraded by Motion Blur and Noise, IEEE Transactions on Automatic Control, vol. AC-27, no. 5, October 1982.

[4] I. M. Rekleities: Optical Flow Recognition from the Power Spectrum of a Single Blurred Image, IEEE International Conference on Image processing(ICIP), 1996.

[5] M. Sakano, N. Suetake, and E. Uchino: A PSF Estimation based on Hough Trasnform Concerning Gradient Vector for Noisy and Motion Blurred Images, IEICE Trans. Inf. \& Syst., vol. E-90D, no. 1, 1 January 2007.

[6] C. Y. Wen and C. H. Lee: Point Spread Fucntions and Their Applications to Forensic Image Restora- 
tion, Forensic Science Journal, vol. 1, no. 1, pp. 15-26, 2002.

[7] R. Lokhande, K. V. Arya, and P. Gupta: Identification of Parameters and Restoration of Motion Blurred Images, Proc. of ACM Symposium on Applied Computing, pp. 301-315, 2006.

[8] M. E. Moghaddam and M. Jamzad: Motion Blur Identification in Noisy Images Using Fuzzy Sets, Proc. IEEE International Symposium on Signal Processing and Information Technology, Athens, Greece, 2005.

[9] W. F. Al Maki, T. Shimahashi, T. Kitagawa, and S. Sugimoto: A New Approach of Blind Image Deconvolution Method without Artifact along the Blurring Paths, Proc. 39th ISCIE International Symposium on Stochastic Systems Theory and Its Application, 2007.

[10] H. C. Andrews and B. R. Hunt: Digital Image Restoration, Prentice Hall, New York, 1977.

[11] M. R. Banham and A. K. Katsaggelos: Digital Image Restoration, IEEE Signal Process. Mag., vol. 14, no. 2, pp. 24-41, 1977.

[12] M. I. Sezan and A. M. Tekalp: Survey of Recent Development in Digital Image Restoration, Optical Engineering, vol. 29, no. 5, pp.393-404, 1990.

[13] S. Fu, Y. Zhang, X. Zhao, Z. Liang, Z. Hou, A. Zou, W. Ye, and L. Bo: Motion Deblurring for a Power Transmission Line Inspection Robot, in D.-S. Huang, K. Li, and G. W. Irwin (Eds): ICIC 2006, LNAI 4114, pp. 866-871, 2006.

[14] H. Özaktaş, M. Ç. Pınar, and M. Akgül: Restoration of Space-Variant Global Blurs Caused by Severe Camera Movements and Coordinate Distortions, J. Opt., pp. 303-310, 1998.

[15] S. C. Som: Analysis of the Effect of Linear Smear on Photographic Images, J. Opt. Soc. Am., vol. 61, pp. 859-864, 1971. 